WINPEC Working Paper Series No.E1809

November 2018

The Projective Core of Symmetric Games with Externalities

Takaaki Abe and Yukihiko Funaki

Waseda INstitute of Political EConomy

Waseda University

Tokyo, Japan 


\title{
The Projective Core of Symmetric Games with Externalities
}

\author{
Takaaki Abe * $\quad$ Yukihiko Funaki ${ }^{\dagger}$
}

October, 2018

\begin{abstract}
The purpose of this paper is to analyze stable distributions and coalition structures in certain economic situations. We consider the projective core as the most myopic core of the cores defined for games with externalities. Although the core is often defined only for the grand coalition, we define the projective core for each coalition structure and apply it to some economic models such as the public goods game, the Cournot oligopoly, and the common pool resource game. Moreover, we formulate the Bertrand oligopoly as a game with externalities. We argue that symmetry is a common property of these models in terms of the partition function. We offer some general results that hold for all symmetric games with externalities and discuss the implications of the economic models. We also provide necessary and sufficient conditions for the projective core of the models to be nonempty.
\end{abstract}

Keywords: Core; Externalities; Oligopoly; Public goods

JEL Classification: C71

\section{Introduction}

Most economic situations simultaneously include both competition and cooperation among the players. A typical example is an oligopoly. The competitive aspects of an oligopoly have been analyzed as strategic interactions in models such as the Cournot oligopoly and the Bertrand oligopoly, where the firms compete on price or quantity to maximize their profits. In contrast, the cooperative aspect has been formulated as a theory of coalition formation in the cooperative game theory. This approach is often used to find a condition that prevents firms from forming a cartel. In addition to oligopoly, there are many economic and political scenarios that contain both strategic interactions and cooperation such as splits and mergers of political parties and the provision of public goods.

Noncooperative game theory mainly describes the strategic aspects, while cooperative game theory focuses on surplus distributions and coalition formation. Although dividing a situation into the two different models allows us to offer specialized analysis, it makes the situation in which both cooperation and competition coexist difficult to formulate. Early attempts to address this problem are Thrall (1961)

* JSPS Research Fellow. Graduate School of Economics, Waseda University. 1-6-1, Nishi-waseda, Shinjuku-ku, Tokyo 169-8050, Japan. E-mail: takatomo3639@asagi.waseda.jp

The author gratefully acknowledges the financial support from the Japan Society for the Promotion of Science (JSPS).

$\dagger$ School of Political Science and Economics, Waseda University. 1-6-1, Nishi-Waseda, Shinjuku-ku, Tokyo 169-8050,

Japan. E-mail: funaki@waseda.jp 
and Thrall and Lucas (1963). These scholars introduced partition function form games to describe the competition among coalitions and cooperation within each coalition.

A partition function form game is also known as a game with externalities. The notion of externalities indicates that the worth of a coalition may depend on a coalition structure of the other coalitions. In the field of games with externalities, the stability of a coalition structure has been considered to be a difficult problem. The difficulty mainly lies in the multiplicity of reactions to a deviation in players. To see this, we consider a coalition structure, say $\mathcal{P}$, that is a partition of a player set $N$, and some players try to deviate from the coalition structure $\mathcal{P}$ by forming their own coalition $S \subseteq N$. Their deviation effects the other players in the following two senses. One is the effect on the coalition structure. Before the deviation, each member of $S$ belongs to his/her own original affiliation in the partition $\mathcal{P}$. However, through the deviation, each member cancels his/her original membership and forms their own new coalition. As a result, their deviation yields a new coalition structure $\mathcal{Q}$. The other effect is a change in the worth of coalitions. We now consider coalition $T$ that has escaped from the structural effect of $S$ 's deviation mentioned above and consists of the same members in both partitions $\mathcal{P}$ and $\mathcal{Q}$. Even the worth of coalition $T$ may change between the two partitions because of the externalities across coalitions. Therefore, if externalities exist, even players who did not participate in the deviation may have an incentive to react to the deviation by reorganizing their own coalition structure. We can consider various formulations of reactions. Some payers may react to a deviation to protect their profit, and others may threaten the deviating players with punishment to prevent them from deviation.

The traditional formulation was initially introduced by von Neumann and Morgenstern (1944) and revisited by Hart and Kurz (1983). These scholars consider that for a deviation to occur in a coalition, the remaining players will dismantle their coalitions, which will be separate into one-person coalitions. Likewise, one can consider the reaction that the remaining players jointly form one large coalition. Another plausible reaction is to reorganize coalitions to minimize the worth of the deviating players. This idea was proposed and studied by Aumann (1967) and Hart and Kurz (1983). In the same manner, Shenoy (1979) proposed maximizing the worth of the deviating players. Bloch and van den Nouweland (2014) proposed a general function called an expectation formation rule that generalizes these reactions and axiomatically characterizes them in a more general framework. ${ }^{* 1}$

The reaction that we consider in this paper can be thought of as "no reaction": the players other than the deviating players do not reorganize their coalitions. We can consider this as the state just after the deviation but before some players react. In this sense, we consider this type of reaction as the most myopic reaction. This reaction was also proposed by Bloch and van den Nouweland (2014), who call it a projection or a projective reaction. Considering its simplicity, one might intuitively conjecture that the projective reaction should be easier to analyze than the other myopic reactions mentioned above. However, the projective reaction has its unique difficulty: the projective coalition structure that results

\footnotetext{
*1 Chwe (1994), Xue (1997), Ray and Vohra (1997), and Diamantoudi and Xue (2003) developed the theory of farsightedness. They consider a sequence of reactions: a deviation causes a reaction, and the reaction causes another reaction, and the sequence of reactions continues. Kóczy (2007) proposed a recursive form of farsightedness and defined recursive optimism and pessimism. Abe (2018) showed that the farsighted stable set and a certain type of myopic core coincide in some coalition structures in symmetric majority games.
} 
from a deviation depends on the original coalition structure from which the coalition deviates. For instance, let $N$ be the player set $\{1,2,3,4,5\}$ and let $\mathcal{P}$ be a partition $\{\{1,2\},\{3,4,5\}\}$. If coalition $S=\{2,3\}$ deviates from $\mathcal{P}$, then the projective coalition structure that results from this deviation is $\{\{1\},\{2,3\},\{4,5\}\}$. On the other hand, each of the myopic reactions mentioned above does not preserve the structural feature of the original partition. For example, if the players in $N \backslash S$ disintegrate into singletons, the resulting coalition structure is $\{S\} \cup\{\{i\} \mid i \in N \backslash S\}$, which does not depend on the original partition $\mathcal{P}$. In other words, the myopic reactions, except for the projection, erase the information on the original coalition structure and simplify the result of the deviation. Most preceding works assume such myopic reactions, which motivates us to use the projective reaction. We formally elaborate on such reactions in Section 6.

As stated in the beginning of this paper, our purpose is to analyze the stability of the distribution and coalition structure in economic scenarios. Famous applications of the partition function form include the Cournot oligopoly, the public goods game (Ray and Vohra, 1997; and Yi, 1997), and the common pool resource game (Funaki and Yamato, 1999). The common property of these games is symmetry. In the class of games with externalities, unlike games without externalities, the notion of symmetry can be plural. In this paper, we employ the symmetry defined by de Clippel and Serrano (2008), which, as we show in the following sections, admits a wider variety of games than the other plausible symmetry concepts. Although the assumption of symmetry is restrictive for games without externalities, it is used for various games describing economic situations in the presence of externalities. In this paper, in addition to the famous applications above, we offer our stability analysis for other economic applications such as Bertrand competition. Moreover, some general propositions that hold for every symmetric game are also offered.

The rest of the paper is organized as follows. In Section 2, we define games with externalities and the projective core. The class of symmetric games and its subclasses are also introduced. In Section 3, we offer some general results that hold for all symmetric games. The public goods game is discussed in this section. The class of the largest coalition games, which includes the Bertrand oligopoly, is analyzed in Section 4. In Section 5, we consider the class of games with partition cardinality properties; this class includes the Cournot oligopoly and the common pool resource game. In Section 6, we discuss other core notions. We conclude this paper with some remarks in Section 7.

\section{Preliminaries}

Let $N=\{1, \ldots, n\}$ be the set of players. A coalition $S$ is a subset of $N$. Let $|S|$ denote the number of players in $S$. For any coalition $S \subseteq N$, a partition of $S$ is given by $\left\{T_{1}, \ldots, T_{h}\right\}$, where $1 \leq h \leq|S|$, $T_{i} \cap T_{j}=\emptyset$ for $i, j=1, \ldots, h(i \neq j), T_{i} \neq \emptyset$ for $i=1, \ldots, h$ and $\bigcup_{i=1}^{h} T_{i}=S$. We typically use $\mathcal{P}, \mathcal{Q}$ to denote a partition. Let $|\mathcal{P}|$ denote the number of coalitions in $\mathcal{P}$. For any $i \in N, \mathcal{P}(i)$ denotes the coalition in $\mathcal{P}$ that contains player $i$. For any coalition $S \subseteq N$, let $\Pi(S)$ be the set of all partitions of $S$. For any $S \subseteq N$, let $\left.\mathcal{P}\right|_{S}$ be the projection of $\mathcal{P}$ on $S$, formally $\left.\mathcal{P}\right|_{S}=\{S \cap C \mid C \in \mathcal{P}, S \cap C \neq \emptyset\}$. Hence, $\left.\mathcal{P}\right|_{S}$ is a partition of $S$. For example, if $\mathcal{P}=\{\{1,2\},\{3,4,5\}\}$ and $S=\{2,3,4\}$, then $\left.\mathcal{P}\right|_{S}=\{\{2\},\{3,4\}\}$. For any partition $\mathcal{P},[|S|]_{S \in \mathcal{P}}$ represents a multiset of cardinalities that admits multiple instances for 
each element. For example, if $\mathcal{P}=\{\{1,2\},\{3,4\},\{5\}\}$, then $[|S|]_{S \in \mathcal{P}}=\{2,2,1\}$.

We define an embedded coalition of $N$ by $(S, \mathcal{P})$ satisfying $\emptyset \neq S \subseteq N, \mathcal{P} \in \Pi(N)$, and $S \in \mathcal{P}$. The set of all embedded coalitions of $N$ is given by

$$
E C(N)=\{(S, \mathcal{P}) \mid \emptyset \neq S \subseteq N, \mathcal{P} \in \Pi(N), \text { and } S \in \mathcal{P}\} .
$$

A game with externalities is a pair $(N, v)$ in which a partition function $v$ assigns a real number to each embedded coalition, namely, $v: E C(N) \rightarrow \mathbb{R}$. By convention, we define $v(\emptyset, \mathcal{P})=0$ for all $\mathcal{P} \in \Pi(N)$. We sometimes call a game with externalities simply a game. Let $\mathcal{G}_{N}$ be the set of all games with externalities whose player set is $N$.

We now introduce the notion of a projective core. In the literature on cooperative game theory, the formation of the grand coalition is often implicitly assumed, and the core is defined for the grand coalition. In this paper, we define the grand coalition for each partition. ${ }^{* 2}$ Let $v \in \mathcal{G}_{N}$. For each $\mathcal{P} \in \Pi(N)$, let $F(v, \mathcal{P})$ be the set of feasible allocations for partition $\mathcal{P}$, formally $F(v, \mathcal{P})=\left\{x \in \mathbb{R}^{N} \mid \sum_{j \in S} x_{j} \leq\right.$ $v(S, \mathcal{P})$ for any $S \in \mathcal{P}\}$. We similarly define the set of efficient allocations for $\mathcal{P}: X(v, \mathcal{P})=\{x \in$ $\mathbb{R}^{N} \mid \sum_{j \in S} x_{j}=v(S, \mathcal{P})$ for any $\left.S \in \mathcal{P}\right\}$. The projective core for $\mathcal{P}$ is defined as follows:

$$
C^{\text {proj }}(v, \mathcal{P})=\left\{x \in X(v, \mathcal{P}) \mid \sum_{j \in S} x_{j} \geq v\left(S,\left.\{S\} \cup \mathcal{P}\right|_{N \backslash S}\right) \text { for any } S \subseteq N\right\} .
$$

We say that a partition has a nonempty projective core if the projective core for $\mathcal{P}$ is nonempty. ${ }^{* 3}$

As described in Section 1, the projective core can be thought of as the most myopic core in the sense of reaction to a deviating coalition. The partition resulting from the deviation of coalition $S$ is given as the combination of coalition $S$ and the projection of $\mathcal{P}$ on the remaining players $N \backslash S$, namely $\left.\mathcal{P}\right|_{N \backslash S}$. Therefore, the resulting partition $\left.\{S\} \cup \mathcal{P}\right|_{N \backslash S}$ depends on $S$ and the original partition $\mathcal{P}$.

Now, we introduce three classes of games.

- Let $\sigma: N \rightarrow N$ be a permutation. We define $\sigma(S)=\{\sigma(i) \mid i \in S\}$, and similarly, $\sigma(\mathcal{P})=\{\sigma(S) \mid S \in$ $\mathcal{P}\}$. A game $v$ is symmetric if for any $\sigma, v(S, \mathcal{P})=v(\sigma(S), \sigma(\mathcal{P}))$. A symmetric game can be also defined as follows: for any $(S, \mathcal{P})$ and $(T, \mathcal{Q})$ in $E C(N)$, if $|S|=|T|$ and $\left[\left|S^{\prime}\right|\right]_{S^{\prime} \in \mathcal{P}}=\left[\left|T^{\prime}\right|\right]_{T^{\prime} \in \mathcal{Q}}$, then $v(S, \mathcal{P})=v(T, \mathcal{Q})$. Let $\mathcal{G}_{N}^{S}$ be the set of symmetric games. ${ }^{* 4}$

- A game $v$ satisfies partition cardinality property (a PCP game) if for any $(S, \mathcal{P})$ and $(T, \mathcal{Q})$, if $|\mathcal{P}|=|\mathcal{Q}|$, then $v(S, \mathcal{P})=v(T, \mathcal{Q})$. Let $\mathcal{G}_{N}^{P C P}$ be the set of PCP games. In a PCP game, $v$ no

*2 Greenberg (1994), Kóczy (2007, 2009), and Kóczy and Lauwers (2004) consider a solution as a pair of payoff distributions and a partition. Our definition also fits this context.

*3 We define the core by inequalities, while one may use domination: for any partition $\mathcal{P} \in \Pi(N)$ and any allocation $x \in X(v, \mathcal{P})$, we say that $(y, \mathcal{Q})$ dominates $x$ if there exists an $S \subseteq N$ such that (i) $y_{j}>x_{j}$ for any $j \in S$, (ii) $\mathcal{Q}=\left.\{S\} \cup \mathcal{P}\right|_{N \backslash S}$, and (iii) $y \in X(v, \mathcal{Q})$. The core for $\mathcal{P}$ is the set of allocations $x$ in $X(v, \mathcal{P})$ that are not dominated by any such $(y, \mathcal{Q})$. Similar to the traditional core for a game without externalities, the inequality core becomes a subset of the dominance core.

*4 We say that a game satisfies strong symmetry if for any $(S, \mathcal{P})$ and $(T, \mathcal{Q})$,

$$
|S|=|T| \text { and }|\mathcal{P}|=|\mathcal{Q}| \Rightarrow v(S, \mathcal{P})=v(T, \mathcal{Q}) .
$$

Note that the class of strong symmetry games $\mathcal{G}_{N}^{S S}$ is different from that of symmetry games: $\mathcal{G}_{N}^{S S} \subseteq \mathcal{G}_{N}^{S}$. 
longer depends on $S$. The number of coalitions in $\mathcal{P}$ determines the worth of the coalitions in $\mathcal{P}$ that is the same for all the coalitions in $\mathcal{P}$.

- A game is called the largest coalition game if there exists a function $f$ such that for any $(S, \mathcal{P}) \in E C(N) \backslash(N,\{N\})$,

$$
\begin{aligned}
& v(S, \mathcal{P})= \begin{cases}f\left(|S|,\left[\left|S^{\prime}\right|\right]_{S^{\prime} \in \mathcal{P}}\right) \geq 0 & \text { if }|S| \geq\left|S^{\prime}\right| \text { for every } S^{\prime} \in \mathcal{P}, \\
0 & ; \text { otherwise }\end{cases} \\
& v(N,\{N\})>0
\end{aligned}
$$

In the largest coalition game, for each partition $\mathcal{P}$, the largest coalition in $\mathcal{P}$ obtains the worth $\left.f\left(|S|,\left[\left|S^{\prime}\right|\right]\right)_{S^{\prime} \in \mathcal{P}}\right)$. Each coalition that is not the largest in $\mathcal{P}$ obtains zero. If some coalitions are equal in the sense of cardinality, each of them obtains the same worth $f\left(|S|,\left[\left|S^{\prime}\right|\right]_{S^{\prime} \in \mathcal{P}}\right)$. Let $\mathcal{G}_{N}^{L C}$ be the set of largest coalition games.

We have $\mathcal{G}_{N}^{P C P} \subseteq \mathcal{G}_{N}^{S}$ and $\mathcal{G}_{N}^{L C} \subseteq \mathcal{G}_{N}^{S}$. Note that each game in the intersection $\mathcal{G}_{N}^{P C P} \cap \mathcal{G}_{N}^{L C}$ is described as follows: $v(N,\{N\})>0$ and $v(S, \mathcal{P})=0$ for any $(S, \mathcal{P}) \in E C(N) \backslash(N,\{N\})$.

In the absence of externalities, the class of symmetric games might be thought of as a small class, while in the presence of externalities, this class contains a wide variety of games. In this paper, we consider a public goods game in the application of a symmetric game. As for the class of largest coalition games, we consider Bertrand competition and a simple example of common goods competition. Moreover, the common pool resource game analyzed by Funaki and Yamato (1999) and the Cournot oligopoly belong to the class of PCP games.

\section{Symmetric games}

We begin with the basic property of a projection.

Lemma 3.1. Let $\mathcal{P} \in \Pi(N)$ and $S^{*} \in \mathcal{P}$. Let $\sigma^{S^{*}}$ satisfy $\sigma(i)=i$ for any $i \in N \backslash S^{*}$. For any $S \subseteq N$,

$$
\left.\left\{\sigma^{S^{*}}(S)\right\} \cup \mathcal{P}\right|_{N \backslash \sigma^{S^{*}}(S)}=\sigma^{S^{*}}\left(\left.\{S\} \cup \mathcal{P}\right|_{N \backslash S}\right) .
$$

Lemma 3.1 shows that the projective partition resulting from the rearranged coalition coincides with the partition that is rearranged after the deviation. Now, we define the equal division of an arbitrary partition: for any game $v \in \mathcal{G}_{N}, E D_{i}(v, \mathcal{P}):=\frac{v(\mathcal{P}(i), \mathcal{P})}{|\mathcal{P}(i)|}$ for every $i \in N$.

The following proposition is an extension of the necessary and sufficient condition for the core of symmetric games without externalities to be nonempty.

Proposition 3.2. Let $v$ be a symmetric game. Let $\mathcal{P} \in \Pi(N)$. Then,

$$
C^{\operatorname{proj}}(v, \mathcal{P}) \neq \emptyset \Longleftrightarrow E D(v, \mathcal{P}) \in C^{\operatorname{proj}}(v, \mathcal{P})
$$

The statement above is equivalent to the following statement: for any $S \subseteq N, \sum_{j \in S} \frac{v(\mathcal{P}(j), \mathcal{P})}{|\mathcal{P}(j)|} \geq$ $v\left(S,\left.\{S\} \cup \mathcal{P}\right|_{N \backslash S}\right)$. Proposition 3.2 is a generalization in the following two senses. The first is regarding the scope of coalition structures. For a game without externalities, the core and its nonemptiness condition are provided for the grand coalition, whereas Proposition 3.2 is a condition for each partition. 
The second is regarding the multiplicity of cores. For games without externalities, the core is uniquely defined. However, in the presence of externalities, multiple reactions are studied because of the deviations, as introduced in Section 1. Multiplicity yields multiple definitions of the core. The pessimistic core, the optimistic core, the disintegrating core, and the merging core are well-known core concepts. ${ }^{* 5}$ It is relatively straightforward to analyze these core concepts because they do not depend on the partition from which a coalition deviates. In contrast, the projective core inherits the feature of projective reaction and, unlike the cores above, depends on the partition for which the projective core is defined.

The following proposition is useful for finding a partition with an empty projective core and will play an important role in analyzing the economic applications.

Proposition 3.3. Let $v$ be a symmetric game. Let $\mathcal{P} \in \Pi(N)$. If there exist coalitions $S, S^{\prime} \in \mathcal{P}$ such that $|S|>\left|S^{\prime}\right|$ and $\frac{v(S, \mathcal{P})}{|S|}>\frac{v\left(S^{\prime}, \mathcal{P}\right)}{\left|S^{\prime}\right|}$, then $C^{\operatorname{proj}}(v, \mathcal{P})=\emptyset$.

Proposition 3.3 is a necessary condition for a partition to have a nonempty core. This condition shows that a partition that contains different sized coalitions must obey a more restrictive condition to have a nonempty projective core. For example, if $\mathcal{P}=\{\{1\},\{2,3\}\}$ and $v(\{1\}, \mathcal{P})=1$, then $v(\{2,3\}, \mathcal{P})$ must be less than or equal to 2 for $\mathcal{P}$ to have a nonempty projective core.

The intuition behind this result lies in the relationship between a deviation and symmetric partitions. For example, we consider a partition $\{\{1,2,3,4\},\{5,6\}\}$. For this partition, there exists a four-person coalition that contains $\{5,6\}$, e.g., $\{3,4,5,6\}$. After such a coalition deviates, its resulting partition, namely $\{\{3,4,5,6\},\{1,2\}\}$, must be symmetric to the initial partition $\{\{1,2,3,4\},\{5,6\}\}$. Similarly, some four-person coalitions exist and deviate from the resulting partition $\{\{3,4,5,6\},\{1,2\}\}$, which leads to another symmetric partition that may be the first partition. In general, for any coalition that contains different sized coalitions, there exists a coalition for which the deviation yields a partition that is symmetric to the original partition. Therefore, some cycles of deviations can be found among symmetric partitions. Such cycles make the projective cores empty for a group of symmetric partitions.

These general conditions become more informative in some specific subclasses. We first analyze the class of public goods games.

\subsection{Public goods games}

Some models of public goods games are provided by Ray and Vohra (1997) and Yi (1997). In this paper, we introduce a slight variation and analyze its projective core.

Consider partition $\mathcal{P} \in \Pi(N)$. In the partition, each coalition produces public goods. For each coalition, the members choose the optimal amount of public goods for them and equally share the corresponding cost. Each member enjoys the same benefit from consuming the public goods and obtains $I(|S|) \geq 0$ as the final payoff, which is calculated as benefit - cost. ${ }^{* 6}$ The benefit of the public goods

\footnotetext{
${ }^{* 5}$ In Hafalir (2007), the disintegrating core is called the $s$-core (singleton-core) and the merging core is called the $m$-core. These concepts are elaborated in Section 6.

*6 Formally, every player $i \in N$ is endowed with one unit of a private good. For each coalition $S \in \mathcal{P}$, every member $i \in S$ contributes $x_{i} \leq 1$. Let $y$ be the level of public goods and $\bar{y}$ be its maximal level. We use $c(y)$ to denote the cost of producing $y$ public goods. Every member of $S$ enjoys the same benefit $b(y)$ from consuming the public goods
} 
produced by coalition $S$ may spill over from $S$ to other coalitions in $\mathcal{P}$. We use $E(|S|) \geq 0$ to denote the external benefit that each player in $N \backslash S$ derives from $S$. Therefore, the members of coalition $S$ also receive some external benefit from other coalitions in $\mathcal{P}$. For example, if we assume that a member of coalition $S$ receives $50 \%$ of the benefits of the public goods produced by another coalition $T$, then, in addition to their own public goods $I(|S|)$, a member of $S$ enjoys $E(|T|):=I(|T|) / 2$ from $T$ as an external benefit. We refer to $I(|S|)$ as an internal effect on each player in $S$ and $E(|S|)$ as an external effect of $S$ on each player in $N \backslash S$.

Formally, $I$ is a function given by $I:\{0, \ldots, n\} \rightarrow \mathbb{R}_{+}$, and similarly, $E:\{0, \ldots, n\} \rightarrow \mathbb{R}_{+}$. We assume that $I(0)=E(0)=0$. We define $\Delta^{I}(k)=I(k)-I(k-1)$ and $\Delta^{E}(k)=E(k)-E(k-1)$ for any $k=1, \ldots, n$ and assume that a marginal internal effect is larger than a marginal external effect:

$$
\Delta^{I}(k)>\Delta^{E}(k)
$$

for any $k=1, \ldots, n$. This assumption indicates that a change in the size of a coalition effects the members more than the nonmembers. For any $\mathcal{P} \in \Pi(N)$ and any $S \in \mathcal{P}$, we define

$$
v(S, \mathcal{P})=|S| \cdot I(|S|)+\sum_{T \in \mathcal{P} \backslash\{S\}}|S| \cdot E(|T|) .
$$

If one considers a model with purely local public goods (or a model without spillovers), then $E(k)=0$ for any $k=1, \ldots, n$.

Lemma 3.4. Let $v$ be a public goods game and let $\mathcal{P} \in \Pi(N)$. If there exist coalitions $S, S^{\prime} \in \mathcal{P}$ such that $|S|>\left|S^{\prime}\right|$, then $C^{\operatorname{proj}}(v, \mathcal{P})=\emptyset$.

The proof is straightforward in view of Proposition 3.3. This lemma implies that for a partition to have a nonempty projective core, every coalition in the partition must have the same cardinality. Moreover, note that Lemma 3.4 does not depend on $I(\cdot)$ and $E(\cdot)$ : the emptiness is valid for any form of internal/external effect functions. What condition guarantees the nonemptiness of the projective core? To determine this, we now focus on convex $I(\cdot)$. The following proposition shows that the convexity gives the grand coalition a nonempty projective core and gives the other partitions empty projective cores.

Proposition 3.5. If $\Delta^{I}(k) \leq \Delta^{I}(k+1)$ for every $k=1, \ldots, n-1$, then $C^{\operatorname{proj}}(v,\{N\}) \neq \emptyset$ and $C^{\text {proj }}(v, \mathcal{P})=\emptyset$ for any $\mathcal{P} \in \Pi(N) \backslash\{N\}$.

Note that spillover effect $E$ is not conditioned in this proposition: $E$ does not influence the nonemptiness of the core under the above condition of $I$. In the presence of a spillover, each member of every coalition benefits from their own public goods and the spillovers from other coalitions. Therefore, one might consider that a partition consisting of multiple coalitions can also be seen as a stable coalition structure. However, Proposition 3.5 shows that the grand coalition, in which there is no such spillover from another coalition, is the only stable coalition structure. This result occurs because of the convexity of $I$. In view of $\Delta^{I}(k)>\Delta^{E}(k)$, the internal effect surpasses the external effect, and the convexity

and equally shares the cost $c(y)$. We set $I(|S|):=\max _{0 \leq y \leq \bar{y}} b(y)-\frac{c(y)}{|S|}$. 
of $I$ benefits a larger coalition, which provides players with a larger incentive to cooperate and jointly produce public goods rather than enjoying the public goods as free riders.

If $I$ is concave, namely, $\Delta^{I}(k) \geq \Delta^{I}(k+1)$ for every $k=1, \ldots, n-1$, and $\Delta^{I}(1) \leq \Delta^{E}(1)$, then the partition of the player set into singletons has a nonempty projective core in a way that is similar to Proposition 3.5. Given the results above, one may consider that if there is $k^{*}$ such that $I(k)$ is convex for $1 \leq k \leq k^{*}-1$ and is concave for $k^{*} \leq k \leq n-1$, then a partition $\mathcal{P}$ satisfying $k^{*}=|S|$ for every $S \in \mathcal{P}$ has a nonempty projective core. However, this conjecture is not true. For example, let $n=10$ and $\mathcal{P}=\left\{S_{1}, S_{2}\right\}$ with $S_{1}=\{1, \ldots, 5\}$ and $S_{2}=\{6, \ldots, 10\}$. Consider $T=\{2, \ldots, 8\}$. We suppose that $I(k)=k$ for $k=1, \ldots, 5, I(k)=5+3 k / 7$ for $k=6, \ldots, 10$, and $E(k)=k / 2$ for every $k=1, \ldots, 10$. Then, $E D_{i}(v, \mathcal{P})=I(5)+E(5)=7.5$, while $v\left(T,\left.\{T\} \cup \mathcal{P}\right|_{N \backslash T}\right)=I(7)+E(1)+E(2)=9.5$. Hence, coalition $T$ has an incentive to deviate from $\mathcal{P}$, and the projective core for the partition $\mathcal{P}$ is empty.

\section{Largest coalition games}

In this section, we consider the class of largest coalition games defined in Section 2. The main purpose of this section is to introduce and analyze the Bertrand oligopoly in partition function form. As preparation, we begin with a general result that is similar to Lemma 3.4.

Corollary 4.1. Let $v$ be a large coalition game and $\mathcal{P} \in \Pi(N)$. If there exist coalitions $S, S^{\prime} \in \mathcal{P}$ such that $|S|>\left|S^{\prime}\right|$, then $C^{\operatorname{proj}}(v, \mathcal{P})=\emptyset$.

Although the resulting appearance is the same as Lemma 3.4, the reasoning behind Corollary 4.1 is different from that of Lemma 3.4. In a large coalition game, the second largest coalition in a partition obtains zero. Therefore, the second condition of Proposition 3.3, namely, $\frac{v(S, \mathcal{P})}{|S|}>\frac{v\left(S^{\prime}, \mathcal{P}\right)}{\left|S^{\prime}\right|}$, always holds for the largest coalition $S$ and another coalition $S^{\prime}$ as long as $v(S, \mathcal{P})$ is positive. If partition $\mathcal{P}$ satisfies $v(S, \mathcal{P})=0$ for all $S \in \mathcal{P}$, then the partition (even if it contains some coalitions with different sizes) does not violate Proposition 3.3. However, all players have an incentive to deviate by forming $N$ and obtain $v(N,\{N\})>0$. Hence, the projective core for partitions satisfying $v(S, \mathcal{P})=0$ for all $S \in \mathcal{P}$ is also empty.

The class of largest coalition games is suitable for describing competition among coalitions. We offer the following simple example.

Example 4.2 (Common goods competition). Consider that some identical and divisible goods are to be distributed. Let $x>0$ be the amount of the goods. For any $\mathcal{P} \in \Pi(N)$, the largest coalition in $\mathcal{P}$ wins all $x$ goods. If some coalitions are equal in the sense of size, they equally share $x$. Therefore, every common goods competition game is a largest coalition game.

In this game, the projective core is empty for all partitions including the grand coalition. To see this, given Corollary 4.1, we focus on partition $\mathcal{P}$, which consists of coalitions of the same size. Let $\mathcal{P}=\left\{S_{1}, \ldots, S_{m}\right\}$, with $\left|S_{1}\right|=\ldots=\left|S_{m}\right|$.

We first consider $\mathcal{P} \neq\{N\}$. Let $T \subseteq N$ be $|T|=\left|S_{1}\right|+1$. Then, $T$ is the largest coalition in 
$\left.\{T\} \cup \mathcal{P}\right|_{N \backslash T}$. Hence, $v\left(T,\left.\{T\} \cup \mathcal{P}\right|_{N \backslash T}\right)=x$, and we have

$$
\sum_{j \in T} E D_{j}(v, \mathcal{P})=|T| \frac{x}{n}<x=v\left(T,\left.\{T\} \cup \mathcal{P}\right|_{N \backslash T}\right) .
$$

Thus, in view of Proposition 3.2, $C^{\text {proj }}(v, \mathcal{P})=\emptyset$.

We now consider $\mathcal{P}=\{N\}$. For every $i \in N$, we have

$$
\sum_{j \in N \backslash\{i\}} E D_{j}(v,\{N\})=(n-1) \frac{x}{n}<x=v(N \backslash\{i\},\{\{i\}, N \backslash\{i\}\}) .
$$

Hence, similarly, $C^{\text {proj }}(v,\{N\})=\emptyset$.

This example shows that the projective core can be empty even for such a simple setting. However, some economic games have a nonempty projective core. We now analyze the Bertrand oligopoly. This is the first formulation of Bertrand competition in partition function form.

\subsection{Bertrand oligopoly with size-dependent cost functions}

Let $c$ be a cost function. Coalition $S$ produces one unit of identical goods with cost $c(|S|)$ : the cost function assigns a real number to each natural number $1, \ldots, n$. We assume that as a coalition becomes larger, the cost monotonically decreases: $c(k)>c(k+1)$ for any $k=1, \ldots, n-1$. We assume $c(n) \geq 0$. The demand function is given by $q(p)$, where $p$ is a price. We assume that $q^{\prime}(p) \leq 0$ and $q(p) \geq 0$ for any $p \geq 0$.

Given partition $\mathcal{P}$, coalitions in $\mathcal{P}$ simultaneously determine the price of the good. The coalition that offers the lowest price, say $p_{*}$, obtains all the demand at that price, $q\left(p_{*}\right)$. Since the cost function depends on the size of the coalition, the largest unique coalition obtains all the demand $q\left(p_{*}\right)$. If some coalitions tie, they receive zero profit, as indicated by the typical Bertrand oligopoly. Let $S$ be the largest unique coalition in $\mathcal{P}$, and let $S^{\prime}$ be the second largest coalition in $\mathcal{P}$. As a result of the Nash equilibrium, coalition $S$ chooses a price so as to maximize its profit:

$$
\max _{p \leq c\left(\left|S^{\prime}\right|\right)} q(p)(p-c(|S|)) .
$$

The largest coalition $S$ must choose $p \leq c\left(\left|S^{\prime}\right|\right)$ to win the price competition and $p>c(|S|)$ to obtain positive profit. We assume that a coalition withdraws from the competition if its profit is zero. ${ }^{* 7} \mathrm{We}$ consider the profit above as the worth of coalition $S$ in $\mathcal{P}$ :

$$
v(S, \mathcal{P})= \begin{cases}\max _{p \leq c\left(\left|S^{\prime}\right|\right)} q(p)(p-c(|S|)) & \text { if }|S|>|T| \text { for every } T \in \mathcal{P} \backslash\{S\} \\ 0 & \text { otherwise. }\end{cases}
$$

If $\mathcal{P}=\{N\}$, the grand coalition $N$ obtains the monopoly profit, where the monopoly price $p^{*}$ solves $q^{\prime}\left(p^{*}\right) \cdot\left(p^{*}-c(n)\right)+q\left(p^{*}\right)=0$. Let $\pi^{*}$ denote monopoly profit. We assume that $\pi^{*}>0$. Note that every Bertrand oligopoly with size-dependent cost functions is a large coalition game. We first obtain the following result.

\footnotetext{
*7 Therefore, if the maximizing price $p$ satisfies $p=c\left(\left|S^{\prime}\right|\right)$, the profit of the largest coalition $S$ is $q(p)(p-c(|S|))=$ $q\left(c\left(\left|S^{\prime}\right|\right)\right)\left(c\left(\left|S^{\prime}\right|\right)-c(|S|)\right)$ and that of the second largest coalition $S^{\prime}$ is zero.
} 
Proposition 4.3. For any $\mathcal{P} \in \Pi(N) \backslash\{N\}, C^{\operatorname{proj}}(v, \mathcal{P})=\emptyset$.

In addition to partitions containing different sized coalitions, some partitions consisting of the same size coalitions also lack projective cores. Therefore, we restrict our attention to the grand coalition to find a nonempty projective core. We first define $h(n)$ as follows:

$$
h(n)= \begin{cases}\frac{n+1}{2} & \text { if } n \text { is odd } \\ \frac{n}{2}+1 & \text { if } n \text { is even. }\end{cases}
$$

For notational simplicity, we simply write $h$ instead of $h(n)$. Now, for cost function $c$ and demand function $q$, we define $d^{c, q}$ as follows: for any $s=1, \ldots, n$,

$$
d^{c, q}(s)=\max _{p \leq c(n-s)} q(p)(p-c(s)) .
$$

Proposition 4.4. The projective core for the grand coalition $C^{\operatorname{proj}}(v,\{N\})$ is nonempty if and only if for every $s=h, \ldots, n-1$,

$$
\frac{d^{c, q(s)}}{s} \leq \frac{\pi^{*}}{n}
$$

Proposition 4.4 is a necessary and sufficient condition for the projective core to be nonempty, which shows that we do not have to check $d^{c, q}(s)$ for $s=1, \ldots, h-1$. This result contributes to the literature on oligopolies in partition function form in three ways.

- One is the generality of the cost function and the demand function. Most preceding works employ a linear demand function and constant marginal costs, while the proposition holds for general demand and cost functions.

- Another novelty is that we formally show that the partitions other than the grand coalition are not stable in terms of the core. The formation of the grand coalition has been often assumed in this context. Proposition 4.3 offers the reasons for this implicit assumption.

- As elaborated in the next section, in a Cournot oligopoly, the projective core of the grand coalition is empty even under the linear setting, and it is also empty for the other partitions. Our result shows that the core can be nonempty for some demand functions and cost functions.

Below, as instances of the third issue discussed above, we offer two numerical examples. In the former example, we show that the grand coalition can have a nonempty core with a simple (linear) demand function and a (linear) cost function. The latter example describes an empty projective core.

Example 4.5. Let $N=\{1,2,3,4,5\}$. Consider $q(p)=\max \{12-p, 0\}$ for $p \in \mathbb{R}^{+}$and $c(s)=6-s$ for $s=1, \ldots, 5$. Note that $h=3$. The monopoly profit $v(N,\{N\})=\pi^{*}$ is given by

$$
\pi^{*}=\max _{p \geq 0} q(p)(p-c(5))=\max _{p \geq 0}(12-p)(p-1)=30.25 .
$$

As Proposition 4.4 shows, it suffices to check $d^{c, q}(3)$ and $d^{c, q}(4)$. We have

$$
\begin{aligned}
& d^{c, q}(3)=\max _{p \leq c(2)} q(p)(p-c(3))=\max _{p \leq 4}(12-p)(p-3)=8 \\
& d^{c, q}(4)=\max _{p \leq c(1)} q(p)(p-c(4))=\max _{p \leq 5}(12-p)(p-2)=21 .
\end{aligned}
$$


Hence, we have $8 / 3=2.666 \ldots<6.05=30.25 / 5$ for $d^{c, q}(3)$ and $21 / 4=5.25<6.05=30.25 / 5$ for $d^{c, q}(4)$, and the projective core for the grand coalition is nonempty. The projective cores for the other partitions are empty, as Proposition 4.3 describes.

Example 4.6. We now consider another (decreasing) cost function with $c(5)=1, c(3)=2$, and $c(2)=7$. We use the same demand function $q(p)=\max \{12-p, 0\}$ for $p \in \mathbb{R}^{+}$. The monopoly profit is the same, $\pi^{*}=30.25$. For $d^{c, q}(3)$, we have

$$
d^{c, q}(3)=\max _{p \leq c(2)} q(p)(p-c(3))=\max _{p \leq 7}(12-p)(p-2)=25
$$

Hence, we have $25 / 3=8.333 \ldots>6.05=30.25 / 5$ for $d^{c, q}(3)$. The necessary and sufficient condition is violated, and the projective core for the grand coalition is empty. Therefore, in this example, every partition has an empty projective core.

\section{Games with partition cardinality properties}

Although each PCP game seems more restrictive than the other symmetric games, this class contains two economic applications: the Cournot oligopoly proposed by Ray and Vohra (1997) and Yi (1997) and the common pool resource game introduced by Funaki and Yamato (1999). ${ }^{* 8}$ The rich results of these two games can be ascribed to the simplification of the partition function. A PCP game $v \in \mathcal{G}_{N}^{P C P}$ is represented by function $f$ such that for any $(S, \mathcal{P}) \in E C(N)$,

$$
f(|\mathcal{P}|)=v(S, \mathcal{P}) .
$$

In other words, the worth of an embedded coalition only depends on the cardinality of the partition. In this section, we use $f$ instead of $v$ to denote a PCP game.

As we have already seen in the previous sections, the projective core often becomes empty for partitions other than the grand coalition because of Proposition 3.3 and its corollaries. Moreover, in some specific instances, the projective core is empty even for the grand coalition. However, in the class of PCP games, the projective core can be nonempty for all partitions. Below, we offer a necessary and sufficient condition for a partition to have a nonempty projective core.

We define PCP game $f$ and partition $\mathcal{P} \in \Pi(N)$. For every $k \geq 0$, we define

$$
g^{f, \mathcal{P}}(k)=\left\{\begin{array}{cl}
(|\mathcal{P}|-k+1) f(|\mathcal{P}|) & k \leq|\mathcal{P}|, \\
\frac{1}{\left|S_{\max }(\mathcal{P})\right|} f(|\mathcal{P}|) & k>|\mathcal{P}|,
\end{array}\right.
$$

where $S_{\text {max }}(\mathcal{P})$ is one of the largest coalitions in $\mathcal{P}:\left|S_{\max }(\mathcal{P})\right| \geq\left|S^{\prime}\right|$ for every $S^{\prime} \in \mathcal{P}$.

Proposition 5.1. Let $f$ be a PCP game and set $\mathcal{P} \in \Pi(N)$. Assume $f(|\mathcal{P}|) \geq 0$. Then,

$$
C^{\operatorname{proj}}(f, \mathcal{P}) \neq \emptyset \Longleftrightarrow f(k) \leq g^{f, \mathcal{P}}(k) \text { for every } k=1, \ldots,|P|+1 .
$$

\footnotetext{
${ }^{* 8}$ Abe and Funaki (2017) also analyze some myopic core notions (see Section 6) of common pool resource games, where the cores are defined for the grand coalition.
} 
Game $f$ can be thought of as a function with one variable, and so is $g^{f, \mathcal{P}}$. The proposition states that the projective core is nonempty if and only if $g^{f \mathcal{P}}$ is located above $f$ for each $k$. Moreover, we can derive some implications from Proposition 5.1.

- If $f(k)$ is nondecreasing in $k$, partition $\{\{i\} \mid i \in N\}$ is the only partition that has a nonempty projective core. If $f(k)$ is nonincreasing in $k$, the nonemptiness of the core is more complicated. The following two games are the PCP games with nonicreasing $f$.

- The common pool resource game has a nonincreasing $f$. Moreover, this game satisfies $k \cdot f(k)>$ $k^{\prime} \cdot f\left(k^{\prime}\right)$ if $k<k^{\prime}$. In view of this inequality, Proposition 5.1 is not satisfied for each partition, which implies that every common pool resource game with $n \geq 3$ has an empty projective core for every partition.

- The Cournot oligopoly is another example. This game is explicitly given as $f(k)=\frac{1}{(k+1)^{2}}$, which immediately violates the necessary and sufficient condition and causes there to be an empty projective core for every partition.

- The observations above show that if $f$ is constant, the partition consisting of singletons $\{\{i\} \mid i \in N\}$ is the only partition that has a nonempty projective core. Moreover, if $f$ is partially constant and increases at some $k^{*}$, a partition whose cardinality is $k^{*}$ may have a nonempty projective core. For example, let $f(k)=c$ for $k \leq k^{*}$ and $f(k)=d$ for $k>k^{*}(c \neq d)$. If some partition $\mathcal{P}$ satisfies $|\mathcal{P}|=k^{*}$ and $c \cdot \frac{1}{\left|S_{\max }(\mathcal{P})\right|} \geq d$, then this partition $\mathcal{P}$ has a nonempty projective core.

- In regards to any single-peaked function, according to our proposition it readily follows that the left side of the peak (namely, the coarse partitions or the partitions for which worth is increasing) lacks a projective core. Similarly, for any single-dipped function, the right side of the dip (namely, the finer partitions) lacks a projective core, except for the partition $\{\{i\} \mid i \in N\}$.

Moreover, the following result follows for "adjacent" partitions.

Corollary 5.2. For any partitions $\mathcal{P}, \mathcal{Q} \in \Pi(N)$ with $|\mathcal{P}|+1=|\mathcal{Q}|$. If $C^{\operatorname{proj}}(f, \mathcal{P}) \neq \emptyset$ and $C^{\text {proj }}(f, \mathcal{Q}) \neq$ $\emptyset$, then $\left|S_{\text {max }}(\mathcal{P})\right|=2$ and $f(|\mathcal{P}|)=2 f(|\mathcal{Q}|)$.

We call the partitions $\mathcal{P}, \mathcal{Q} \in \Pi(N)$, satisfying $|\mathcal{P}|+1=|\mathcal{Q}|$ adjacent partitions (in the sense of cardinality). This corollary indicates that the adjacent partitions seldom have nonempty projective cores simultaneously. For example, consider partition $\mathcal{Q}$. Assume that the core for $\mathcal{Q}$ is nonempty. Then, we have to find partition $\mathcal{P}$, which consists of only two-person coalitions and one-person coalitions and satisfies $|\mathcal{P}|+1=|\mathcal{Q}|$. Furthermore, the worth of partition $\mathcal{P}, f(|\mathcal{P}|)$, must be exactly equal to $2 f(|\mathcal{Q}|)$. In most games, such a partition $\mathcal{P}$ does not exist, which shows how difficult it can be for two adjacent partitions to simultaneously have nonempty projective cores.

\section{Other core concepts}

This paper analyzes the projective core of symmetric games in the presence of externalities. We examine the projective core of each partition because the projective partition resulting from a deviating coalition depends on the feature of the original partition from which the coalition deviates. This property 
means that unlike the cores based on other forms of reactions, the projective core cannot be reduced to games without externalities, i.e., coalition function form games. To show this, we provide a short introduction on expectation formation rules.

The concept of the expectation formation rule was introduced by Bloch and van den Nouweland (2014). Fix player set $N$. An expectation formation rule is a function given by $\psi(S, v, \mathcal{P}) \in \Pi(N)$ satisfying $S \in \psi(S, v, \mathcal{P})$ : it assigns a partition of $N$ containing $S$ to each $(S, v, \mathcal{P}) \in 2^{N} \backslash\{\emptyset\} \times \mathcal{G}_{N} \times \Pi(N)$. Note that the input is not an embedded coalition: $S$ is not necessarily in $\mathcal{P}$. We formally define the reactions mentioned in Section 1 and the projective reaction as expectation formation rules.

The pessimistic expectation: $\psi(S, v, \mathcal{P})=\underset{\mathcal{P}^{\prime}: S \in \mathcal{P}^{\prime} \in \Pi(N)}{\arg \min } v\left(S, \mathcal{P}^{\prime}\right)$

The optimistic expectation: $\psi(S, v, \mathcal{P})=\underset{\mathcal{P}^{\prime}: S \in \mathcal{P}^{\prime} \in \Pi(N)}{\arg \max } v\left(S, \mathcal{P}^{\prime}\right)$.

The disintegrating expectation: $\psi(S, v, \mathcal{P})=\{S\} \cup\{\{i\} \mid i \in N \backslash S\}$.

The merging expectation: $\psi(S, v, \mathcal{P})=\{S, N \backslash S\}$.

The projective expectation: $\psi(S, v, \mathcal{P})=\left.\{S\} \cup \mathcal{P}\right|_{N \backslash S}$.

It can be seen that the pessimistic, optimistic, disintegrating, and merging expectations are independent from input $\mathcal{P}$. Because of this independence, each of these four rules is represented by $\psi(s, v)$ and reduces a partition function $v$ to a coalition function $v^{\psi}$ as follows:

$$
v^{\psi}(S):=v(S, \psi(S, v)) \text { for all } S \subseteq N .
$$

The core also follows this simplification and satisfies

$$
C\left(v^{\psi}\right)=C^{\psi}(v)
$$

where $C^{\psi}(v)=\left\{x \in \mathbb{R}^{N} \mid \sum_{j \in S} x_{j} \geq v(S, \psi(S, v)) \forall S \subseteq N, \sum_{j \in N} x_{j}=v(N,\{N\}\}\right.$. Below, by simplifying the models studied in this paper, we can provide them in coalition function form. In particular, we focus on the pessimistic game $v^{\text {pes }}$ and the optimistic game $v^{\text {opt }}$ because the optimistic (pessimistic) core is the smallest (largest) core of all types of cores for games with externalities. Therefore, it holds that $C^{\mathrm{opt}}(v, \mathcal{P}) \subseteq C^{\mathrm{proj}}(v, \mathcal{P}) \subseteq C^{\mathrm{pes}}(v, \mathcal{P})$ for any $v \in \mathcal{G}_{N}$ and $\mathcal{P} \in \Pi(N)$.

- The PCP games: If game $f$ is nondecreasing, the pessimistic game is very simple: $v^{\text {pes }}(S)=f(2)$ for any $S \subsetneq N$ and $v^{\text {pes }}(N)=f(1)$. The optimistic game is $v^{\text {opt }}(S)=f(n-s+1)$ for any $S \subseteq N$. The intuition behind this is regarding nonnegative externalities. A game is said to have nonnegative externalities if $v\left(S,\left\{T_{1} \cup T_{2}\right\} \cup \mathcal{P}^{\prime}\right) \geq v\left(S,\left\{T_{1}, T_{2}\right\} \cup \mathcal{P}^{\prime}\right)$ for any $\mathcal{P}^{\prime} \in \Pi\left(N \backslash\left(T_{1} \cup T_{2}\right)\right)$ and $S \in \mathcal{P}^{\prime}$, which means that a merger of two coalitions provides the other coalitions with a "good" effect. If $f$ is nonincreasing, then the game has nonpositive externalities and the formulas are simply swapped between $v^{\text {pes }}$ and $v^{\text {opt }}$.

- The Bertrand oligopoly: The pessimistic game is generally given as follows: for any $S \subseteq N$,

$$
v^{\mathrm{pes}}(S)= \begin{cases}\max _{p \leq c(n-s)} q(p)(p-c(s)) & \text { if } s \geq h, \\ 0 & \text { if } s \leq h-1 .\end{cases}
$$

Note that the Bertrand oligopoly has nonpositive externalities, which means that the more rival firms grow larger, the less our firm earns. Hence, the pessimistic deviating players anticipate that 
all the rival firms will merge with each other and form a large firm. In contrast, if deviating players have an optimistic expectation, they expect that the other firms will separate into singletons. Therefore, the optimistic game is given by

$$
v^{\mathrm{opt}}(S)= \begin{cases}\max _{p \leq c(1)} q(p)(p-c(s)) & \text { if } s \geq 2, \\ 0 & \text { if } s=1 .\end{cases}
$$

- The public goods game: Our public goods game is defined with general functions $I$ and $E$. In particular, function $E$ determines the externalities. If $E$ is superadditive, namely $E\left(k+k^{\prime}\right) \geq$ $E(k)+E\left(k^{\prime}\right)$ for any $k, k^{\prime}=0, \ldots, n$ with $k+k^{\prime} \leq n$, then the game obtains nonnegative externalities. The pessimistic game is simply defined as

$$
v^{\mathrm{pes}}(S)=s \cdot I(s)+s(n-s) \cdot E(1),
$$

while the optimistic game is

$$
v^{\mathrm{opt}}(S)=s \cdot I(s)+s \cdot E(n-s) .
$$

In the case with subadditive $E$, these formulas are swapped.

Even after reducing to a game without externalities, our core is defined over all coalition structures. Therefore, even if the traditional core, which is only defined for the grand coalition, is empty, some core allocations may exist for other coalition structures. Our results for the projective core are useful to "detect" such core allocations. Since the pessimistic core is a superset of the projective core, if the projective core is nonempty, then the pessimistic core is also nonempty. Similarly, if a partition has an empty projective core, there is no optimistic core allocation in the partition.

\section{Concluding remarks}

\subsection{Value concepts}

In addition to the core concepts discussed in this paper, many value concepts are also proposed for games with externalities. Myerson (1977), Bolger (1989), Macho-Stadler et al. (2007), Albizuri et al. (2005), and de Clippel and Serrano (2008) defined these value concepts. Each of concept can be seen as a generalization of the Shapley value to games with externalities and hence satisfies efficiency for the grand coalition. Therefore, each value concept distributes the surplus of the grand coalition and is not defined for other coalition structures. This does not match the core concept studied in this paper. A general question arises: How can we define a value concept for each coalition structure? One possible approach is adopting a value concept for games with coalition structures (henceforth, a CS-value and a CS-game). Since a CS-game is a pair of games without externalities and a partition, we may need to use an expectation function as mentioned in the previous section to reduce games with externalities. Fixing a game (without externalities), we apply a CS-value to every coalition. As a result, every partition has one allocation. Considering that each core allocation satisfies coalition efficiency, namely, $\sum_{j \in S} x_{j}=v(S, \mathcal{P})$ for every $S \in \mathcal{P}$, the allocation derived from a CS-value should also satisfy coalition efficiency. We can use, for example, the Aumann-Drèze value, the Casajus value, the Wiese value, and the nucleolus to 
achieve coalition efficiency. ${ }^{* 9}$ This application allows us to argue which value lies in our core. Given that a symmetric game has an advantage of general coincidence between the equal division $E D(v, \mathcal{P})$ and each of the value concepts, replacing $E D(v, \mathcal{P})$ with each value concept yields another expression of the condition that the core will be nonempty in a symmetric game (Proposition 3.2).

\subsection{Other symmetry definitions}

In this paper, we define a symmetric game as a game $v$ satisfying $v(S, \mathcal{P})=v(\sigma(S), \sigma(\mathcal{P})$ ) for any permutation $\sigma$. In addition, we also define strong symmetry $(\mathrm{SS})$ : for any $(S, \mathcal{P})$ and $(T, \mathcal{Q})$, $|S|=|T|$ and $|\mathcal{P}|=|\mathcal{Q}| \Rightarrow v(S, \mathcal{P})=v(T, \mathcal{Q})$. In view of the definition of strong symmetry, one can immediately define two weaker variations, say coalition symmetry and partition symmetry, as follows. A game $v$ satisfies coalition symmetry $(\mathrm{CS})$ if for any $(S, \mathcal{P})$ and $(T, \mathcal{Q}),|S|=|T|$ and $\mathcal{P}=$ $\mathcal{Q} \Rightarrow v(S, \mathcal{P})=v(T, \mathcal{Q})$. A game $v$ satisfies partition symmetry $(\mathrm{PS})$ if for any $(S, \mathcal{P})$ and $(T, \mathcal{Q})$, $S=T$ and $|\mathcal{P}|=|\mathcal{Q}| \Rightarrow v(S, \mathcal{P})=v(T, \mathcal{Q})$. From the definitions above, it readily follows that

- $\mathrm{SS} \Rightarrow$ Symmetry $\Rightarrow \mathrm{CS}$,

- $\mathrm{SS} \Rightarrow \mathrm{PS}$.

Note that symmetry does not imply PS since another equivalent definition of symmetry is given as $|S|=|T|$ and $\left[\left|S^{\prime}\right|\right]_{S^{\prime} \in \mathcal{P}}=\left[\left|T^{\prime}\right|\right]_{T^{\prime} \in \mathcal{Q}} \Rightarrow v(S, \mathcal{P})=v(T, \mathcal{Q})$. CS requires $\mathcal{P}$ to completely coincide with $\mathcal{Q}$, which simply means that two coalitions with the same size have the same worth in a partition. However, as described in the proofs, this is not sufficient for the proofs of the propositions in Section 3 to hold.

\section{Appendix}

\section{Proof of Lemma 3.1}

Proof. First, we have

$$
\sigma^{S^{*}}\left(\left.\{S\} \cup \mathcal{P}\right|_{N \backslash S}\right)=\left\{\sigma^{S^{*}}(S)\right\} \cup \sigma^{S^{*}}\left(\left.\mathcal{P}\right|_{N \backslash S}\right)
$$

We focus on $\sigma^{S^{*}}\left(\left.\mathcal{P}\right|_{N \backslash S}\right)$. Then, we have

$$
\begin{aligned}
\sigma^{S^{*}}\left(\left.\mathcal{P}\right|_{N \backslash S}\right) & =\sigma^{S^{*}}(\{(N \backslash S) \cap C \mid C \in \mathcal{P}\}) \\
& =\left\{\sigma^{S^{*}}((N \backslash S) \cap C) \mid C \in \mathcal{P}\right\} \\
& =\left\{\sigma^{S^{*}}(N \backslash S) \cap \sigma^{S^{*}}(C) \mid C \in \mathcal{P}\right\} \\
& =\left\{\left(N \backslash \sigma^{S^{*}}(S)\right) \cap \sigma^{S^{*}}(C) \mid C \in \mathcal{P}\right\} \\
& \stackrel{S^{*} \in \mathcal{P}}{=}\left\{\left(N \backslash \sigma^{S^{*}}(S)\right) \cap C \mid C \in \mathcal{P}\right\} \\
& =\left.\mathcal{P}\right|_{N \backslash \sigma^{S^{*}}(S)} .
\end{aligned}
$$

Hence, (A.1) is equal to $\left.\left\{\sigma^{S^{*}}(S)\right\} \cup \mathcal{P}\right|_{N \backslash \sigma^{*}}(S)$.

\footnotetext{
${ }^{* 9}$ See Casajus (2009) for detailed definitions.
} 


\section{Proof of Proposition 3.2}

Proof. $\Leftarrow$ is clear. Below, we show $\Rightarrow$. Let $\mathcal{P}=\left\{S_{1}, \ldots, S_{|\mathcal{P}|}\right\}$. For every $S \in \mathcal{P}$, let $\sigma^{S}$ satisfy $\sigma^{S}(i)=i$ for every $i \in N \backslash S$, i.e., $\sigma^{S}$ denotes a permutation only for the members in $S$. Let $x \in C^{\operatorname{proj}}(v, \mathcal{P})$. From the definition of $C^{\text {proj }}$, it follows that

$$
\begin{aligned}
& \sum_{j \in S} x_{j} \geq v\left(S,\left.\{S\} \cup \mathcal{P}\right|_{N \backslash S}\right) \text { for any } S \subseteq N, \\
& \sum_{j \in S} x_{j}=v(S, \mathcal{P}) \text { for any } S \in \mathcal{P} .
\end{aligned}
$$

For any $\sigma$, we define $\sigma x_{i}:=x_{\sigma(i)}$ for any $i \in N$. Then, for any $S \subseteq N$, we have

$$
\begin{aligned}
& \sum_{j \in S} \sigma^{S_{1}} x_{j}=\sum_{j \in S} x_{\sigma^{S_{1}}(j)} \\
&=\sum_{j \in \sigma^{S_{1}}(S)} x_{j} \\
& \stackrel{(A .2)}{\geq} v\left(\sigma^{S_{1}}(S),\left.\left\{\sigma^{S_{1}}(S)\right\} \cup \mathcal{P}\right|_{N \backslash \sigma^{S_{1}}(S)}\right) \\
& \stackrel{\mathrm{Lma}^{\prime} .1}{=} v\left(\sigma^{S_{1}}(S), \sigma^{S_{1}}\left(\left.\{S\} \cup \mathcal{P}\right|_{N \backslash S}\right)\right) \\
& \stackrel{v \in \mathcal{G}_{N}^{S}}{=} v\left(S,\left.\{S\} \cup \mathcal{P}\right|_{N \backslash S}\right) .
\end{aligned}
$$

Similarly, for every $S \in \mathcal{P}$,

$$
\sum_{j \in S} \sigma^{S_{1}} x_{j} \stackrel{(A .3), \operatorname{Lma3} .1, v \in \mathcal{G}_{N}^{S}}{=} v(S, \mathcal{P}) .
$$

Hence, $\sigma^{S_{1}} x \in C^{\operatorname{proj}}(v, \mathcal{P})$. This holds for every permutation $\sigma^{S_{1}}$. Note that there are $\left|S_{1}\right|$ ! permutations that arrange the members in $S_{1}$. We denote the set of the $\left|S_{1}\right|$ ! permutations by $A^{S_{1}}$. We define $y:=\frac{1}{\left|S_{1}\right| !} \sum_{\sigma^{S_{1}} \in A^{S_{1}}} \sigma^{S_{1}} x$. For any player $i \in S_{1}$, we have

$$
\begin{aligned}
y_{i} & =\frac{1}{\left|S_{1}\right| !} \sum_{\sigma^{S_{1} \in A^{S_{1}}}} \sigma^{S_{1}} x_{i} \\
& =\frac{1}{\left|S_{1}\right| !}\left(\left|S_{1}\right|-1\right) !\left(\sum_{j \in S_{1}} x_{j}\right) \\
& \stackrel{(\text { A.3) }}{=} \frac{1}{\left|S_{1}\right| !}\left(\left|S_{1}\right|-1\right) ! v\left(S_{1}, \mathcal{P}\right) \\
& =\frac{v\left(S_{1}, \mathcal{P}\right)}{\left|S_{1}\right|} .
\end{aligned}
$$

Hence, allocation $y$ is given as

$$
y_{i}:=\left\{\begin{array}{ll}
\frac{v\left(S_{1}, \mathcal{P}\right)}{\left|S_{1}\right|} & \text { for every } i \in S_{1}, \\
x_{i} & \text { for every } i \in N \backslash S_{1},
\end{array} \quad \text { for every } i \in N\right.
$$

and, in view of the convexity of the core, belongs to $C^{\text {proj }}(v, \mathcal{P})$, namely, with slightly abusing the notation, $y=\left(E D^{S_{1}}, x^{S_{2}}, \ldots, x^{S_{|\mathcal{P}|}}\right) \in C^{\operatorname{proj}}(v, \mathcal{P})$. We repeat this process for each $S_{2}, \ldots, S_{|\mathcal{P}|}$ and obtain $E D(v, \mathcal{P}) \in C^{\operatorname{proj}}(v, \mathcal{P})$. 


\section{Proof of Proposition 3.3}

Proof. In view of Proposition 3.2, we show that $E D(v, \mathcal{P}) \notin C^{\operatorname{proj}}(v, \mathcal{P})$. Let $S$ and $S^{\prime}$ in $\mathcal{P}$ satisfy

$$
\begin{gathered}
|S|>\left|S^{\prime}\right|, \\
\frac{v(S, \mathcal{P})}{|S|}>\frac{v\left(S^{\prime}, \mathcal{P}\right)}{\left|S^{\prime}\right|} .
\end{gathered}
$$

In view of (A.4), there exists a coalition $T \subseteq N$ such that

$$
\begin{aligned}
|T| & =|S|, \\
T & =S^{\prime} \cup R \text { for some } \emptyset \neq R \subsetneq S .
\end{aligned}
$$

We have

$$
\begin{aligned}
|S \backslash T| & \stackrel{(A .7)}{=}\left|S \backslash\left(S^{\prime} \cup R\right)\right| \\
& =|S \backslash R| \\
& =|S|-|R| \\
& \stackrel{(A .7)}{=}|S|-\left(|T|-\left|S^{\prime}\right|\right) \\
& \stackrel{(A .6)}{=}\left|S^{\prime}\right| .
\end{aligned}
$$

We have

$$
\begin{aligned}
& \left.v\left(T,\left.\{T\} \cup \mathcal{P}\right|_{N \backslash T}\right) \quad \stackrel{(A .7)}{=} \quad v\left(T,\left.\{T, S \backslash T\} \cup \mathcal{P}\right|_{N \backslash(T \cup S)}\right\}\right) \\
& v \in \mathcal{G}_{N}^{S}, \stackrel{(A .6),(A .8)}{=} v\left(S,\left.\left\{S, S^{\prime}\right\} \cup \mathcal{P}\right|_{N \backslash\left(S \cup S^{\prime}\right)}\right) \\
& =v(S, \mathcal{P}) \text {. }
\end{aligned}
$$

Hence, we obtain

$$
\begin{aligned}
\sum_{j \in T} E D_{j}(v, \mathcal{P}) & \stackrel{(A .7)}{=}|R| \frac{v(S, \mathcal{P})}{|S|}+\left|S^{\prime}\right| \frac{v\left(S^{\prime}, \mathcal{P}\right)}{\left|S^{\prime}\right|} \\
& \stackrel{(A .5)}{<}|R| \frac{v(S, \mathcal{P})}{|S|}+\left|S^{\prime}\right| \frac{v(S, \mathcal{P})}{|S|} \\
& \stackrel{(A .7)}{=}|T| \frac{v(S, \mathcal{P})}{|S|} \\
\stackrel{(A .6),(A .9)}{=} & |T| \frac{v\left(T,\left.\{T\} \cup \mathcal{P}\right|_{N \backslash T}\right)}{|T|} \\
= & v\left(T,\left.\{T\} \cup \mathcal{P}\right|_{N \backslash T}\right) .
\end{aligned}
$$

Thus, $E D(v, \mathcal{P}) \notin C^{\operatorname{proj}}(v, \mathcal{P})$.

\section{Proof of Lemma 3.4}

Proof. Let $S, S^{\prime} \in \mathcal{P}$ with $|S|>\left|S^{\prime}\right|$. We have

$$
\frac{v(S, \mathcal{P})}{|S|}=I(|S|)+\sum_{T \in \mathcal{P} \backslash\{S\}} E(|T|) \text { and } \frac{v\left(S^{\prime}, \mathcal{P}\right)}{\left|S^{\prime}\right|}=I\left(\left|S^{\prime}\right|\right)+\sum_{T \in \mathcal{P} \backslash\left\{S^{\prime}\right\}} E(|T|) .
$$


Hence

$$
\begin{aligned}
\frac{v(S, \mathcal{P})}{|S|}-\frac{v\left(S^{\prime}, \mathcal{P}\right)}{\left|S^{\prime}\right|} & =I(|S|)-I\left(\left|S^{\prime}\right|\right)-\left[E(|S|)-E\left(\left|S^{\prime}\right|\right)\right] \\
& =\sum_{k=\left|S^{\prime}\right|+1}^{|S|} \Delta^{I}(k)-\sum_{k=\left|S^{\prime}\right|+1}^{|S|} \Delta^{E}(k) \\
& >0 .
\end{aligned}
$$

Proposition 3.3 completes the proof.

\section{Proof of Proposition 3.5}

Proof. We first show that $C^{\operatorname{proj}}(v, \mathcal{P})=\emptyset$ for any $\mathcal{P} \in \Pi(N) \backslash\{N\}$. In view of Lemma 3.4, consider $\mathcal{P} \neq\{N\}$ satisfying $k^{*}:=\left|S^{\prime}\right|$ for every $S^{\prime} \in \mathcal{P}$. We have $E D_{i}(v, \mathcal{P})=I\left(k^{*}\right)+(|\mathcal{P}|-1) E\left(k^{*}\right)$ for every $i \in N$. Consider $S \subseteq N$ with $|S|=k^{*}+1$. We have

$$
\begin{aligned}
& v\left(S,\left\{S,\left.\mathcal{P}\right|_{N \backslash S}\right\}\right)-\sum_{j \in S} E D_{j}(v, \mathcal{P}) \\
= & s\left[I\left(k^{*}+1\right)+E\left(k^{*}-1\right)+(|\mathcal{P}|-2) E\left(k^{*}\right)\right]-s\left[I\left(k^{*}\right)+(|\mathcal{P}|-1) E\left(k^{*}\right)\right] \\
= & s\left[I\left(k^{*}+1\right)-I\left(k^{*}\right)-\left(E\left(k^{*}\right)-E\left(k^{*}-1\right)\right)\right] \\
= & s\left[\Delta^{I}\left(k^{*}+1\right)-\Delta^{E}\left(k^{*}\right)\right] \\
> & s\left[\Delta^{I}\left(k^{*}+1\right)-\Delta^{I}\left(k^{*}\right)\right] \\
\geq & 0 .
\end{aligned}
$$

Hence, $E D(v, \mathcal{P}) \notin C^{\operatorname{proj}}(v, \mathcal{P})$. From Proposition $3.2, C^{\operatorname{proj}}(v, \mathcal{P})=\emptyset$ follows.

Now, we show that $C^{\operatorname{proj}}(v,\{N\}) \neq \emptyset$. For any $i \in N, E D_{i}(v,\{N\})=I(n)$. For any $S \subseteq N$, $v(S,\{S, N \backslash S\})=s[I(s)+E(n-s)]$. Hence, for any $S \subseteq N$, we have

$$
\begin{aligned}
\sum_{j \in S} E D_{j}(v, \mathcal{P})-v(S,\{S, N \backslash S\}) & =s[I(n)-I(s)-E(n-s)] \\
& =s[I(n)-I(s)-(E(n-s)-0)] \\
& =s\left[\sum_{k=s+1}^{n} \Delta^{I}(k)-\sum_{k=1}^{n-s} \Delta^{E}(k)\right] \\
& \geq s\left[\sum_{k=1}^{n-s} \Delta^{I}(k)-\sum_{k=1}^{n-s} \Delta^{E}(k)\right] \\
& >0,
\end{aligned}
$$

which implies $C^{\operatorname{proj}}(v,\{N\}) \neq \emptyset$.

\section{Proof of Proposition 4.3}

Proof. In view of Corollary 4.1, if a partition contains some coalitions whose sizes are mutually different, then the projective core for the partition is empty. Hence, consider $\mathcal{P}$ satisfying $|S|=\left|S^{\prime}\right|$ for any $S, S^{\prime} \in \mathcal{P}$. This implies $c(|S|)=c\left(\left|S^{\prime}\right|\right)$ for any $S, S^{\prime} \in \mathcal{P}$. Hence, each coalition obtains profit zero in $\mathcal{P}$, 
which implies that all coalitions have an incentive to form the grand coalition and share the monopoly profit $\pi^{*}>0$.

\section{Proof of Proposition 4.4}

Proof. If-part: From (4.1), it follows that for any coalition $S$ with $|S|=: s$, we have

$$
v(S,\{S, N \backslash S\})= \begin{cases}\max _{p \leq c(n-s)} q(p)(p-c(s)) & \text { for } h \leq s \leq n-1 \\ 0 & \text { for } 1 \leq s \leq h-1 .\end{cases}
$$

Hence, for any $S \subseteq N$ with $h \leq s \leq n-1$, we obtain

$$
\sum_{j \in S} E D_{j}(v, N)=s \frac{\pi^{*}}{n} \geq d^{c, q}(s) \stackrel{(4.2)(A .10)}{=} v(S,\{S, N \backslash S\})
$$

For any $S \subseteq N$ with $1 \leq s \leq h-1$, we have

$$
\sum_{j \in S} E D_{j}(v, N)=s \frac{\pi^{*}}{n} \geq 0 \stackrel{(A .10)}{=} v(S,\{S, N \backslash S\})
$$

Thus, $E D(v, N) \in C^{\operatorname{proj}}(v,\{N\})$.

Only-if-part: From Proposition 3.2, it follows that $E D(v, N) \in C^{\operatorname{proj}}(v,\{N\})$. We have

$$
v(S,\{S, N \backslash S\}) \leq|S| \frac{\pi^{*}}{n}
$$

for every $S \subseteq N$. Hence, in view of (4.2) and (A.10), for every $s=h, \ldots, n-1$,

$$
d^{c, q(s)} \leq s \frac{\pi^{*}}{n} .
$$

\section{Proof of Proposition 5.1}

We fix a PCP game $f$ and a partition $\mathcal{P} \in \Pi(N)$. For convenience, we offer the definition of $g^{f, \mathcal{P}}$ again:

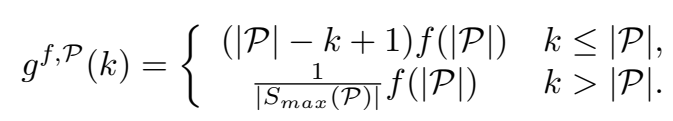

Proof. $\Leftarrow$ : Assume that $C^{\operatorname{proj}}(v, \mathcal{P})=\emptyset$. Since $f(|\mathcal{P}|) \geq 0, X_{+}(v, \mathcal{P}):=\left\{x \in X(v, \mathcal{P}) \mid x_{j} \geq 0\right.$ for any $j \in$ $N\}$ is not empty. Let $x \in X_{+}(v, \mathcal{P})$. As the projective core for $\mathcal{P}$ is empty, there exists a coalition $S \subseteq N$ such that

$$
\sum_{j \in S} x_{j}<v\left(S,\left.\{S\} \cup \mathcal{P}\right|_{N \backslash S}\right)=f(k),
$$

where $k=\left|\left\{S,\left.\mathcal{P}\right|_{N \backslash S}\right\}\right|$. For the coalition $S$, define $\mathcal{T}^{S}=\left\{T \in \mathcal{P}|T \in \mathcal{P}|_{S}\right\}$. Note that

$$
\left|\mathcal{T}^{S}\right|=|\mathcal{P}|-k+1
$$


If $\left|\mathcal{T}^{S}\right| \geq 1$, then $k \stackrel{(A .13)}{\leq}|\mathcal{P}|$. We have

$$
\begin{gathered}
\sum_{j \in S} x_{j} \stackrel{x \in X(v, \mathcal{P})}{=}\left|\mathcal{T}^{S}\right| f(|\mathcal{P}|)+\sum_{j \in S \backslash\left(\cup_{T \in \mathcal{T}} T\right)} x_{j} \\
\stackrel{x_{j} \geq 0}{\geq}\left|\mathcal{T}^{S}\right| f(|\mathcal{P}|) \\
\stackrel{(A .13)}{=}(|\mathcal{P}|-k+1) f(|\mathcal{P}|) .
\end{gathered}
$$

Hence, from (A.12), it follows that

$$
f(k)>(|\mathcal{P}|-k+1) f(|\mathcal{P}|) .
$$

However, in view of (A.11), for any $k \leq|\mathcal{P}|, f(k) \leq g^{f, \mathcal{P}}(k)$ implies that $f(k) \leq(|\mathcal{P}|-k+1) f(|\mathcal{P}|)$. This is a contradiction.

Next, if $\left|\mathcal{T}^{S}\right|=0$, then $k=|\mathcal{P}|+1$. Since for any $x \in X_{+}(v, \mathcal{P})$ there exists $S \subseteq N$ satisfying (A.12), such a coalition $S$ also exists for the equal division $E D(f, \mathcal{P}) \in X_{+}(v, \mathcal{P})$, i.e., $E D_{j}(f, \mathcal{P})=\frac{f(|\mathcal{P}|)}{|\mathcal{P}(j)|}$ for every $j \in N$. Note that $E D_{j}(f, \mathcal{P}) \geq 0$ for every $j \in N$ as $f(|\mathcal{P}|) \geq 0$. Hence, there exists a player $i \in S$ such that

$$
E D_{i}(f, \mathcal{P}) \leq \sum_{j \in S} E D_{j}(f, \mathcal{P}) \stackrel{(A .12)}{<} f(k)
$$

Moreover,

$$
E D_{i}(f, \mathcal{P})=\frac{f(|\mathcal{P}|)}{|\mathcal{P}(i)|} \geq \frac{f(|\mathcal{P}|)}{\left|S_{\max }(\mathcal{P})\right|} .
$$

Hence, we have $\frac{f(|\mathcal{P}|)}{\left|S_{\max }(\mathcal{P})\right|}<f(k)$. However, in view of $(\mathrm{A} .11)$, for any $k>|\mathcal{P}|, f(k) \leq g^{f, \mathcal{P}}(k)$ implies that $f(k) \leq \frac{f(|\mathcal{P}|)}{\left|S_{\max }(\mathcal{P})\right|}$. This is a contradiction.

$\Rightarrow$ : We show that if there exists $k \in\{1, \ldots,|\mathcal{P}|+1\}$ such that $f(k)>g^{f, \mathcal{P}}(k)$, then $C^{\text {proj }}(v, \mathcal{P})=\emptyset$. Assume $x \in C^{\operatorname{proj}}(v, \mathcal{P})$. If $k \leq|\mathcal{P}|$, we have $f(k) \stackrel{(A .11)}{>}(|\mathcal{P}|-k+1) f(|\mathcal{P}|) \stackrel{x \in X(v, \mathcal{P})}{=} \sum_{a=1}^{|\mathcal{P}|-k+1} \sum_{j \in S_{a}} x_{j}$, where $S_{1}, \ldots, S_{|\mathcal{P}|-k+1}$ are arbitrary $|\mathcal{P}|-k+1$ coalitions in $\mathcal{P}$. Hence, $|\mathcal{P}|-k+1$ coalitions in $\mathcal{P}$ have an incentive to jointly deviate by merging and obtain $f(k)$ in total after the deviation. If $k>|\mathcal{P}|$, then (A.11) implies $f(k)>\frac{1}{\left|S_{\max }(\mathcal{P})\right|} f(|\mathcal{P}|)$. Moreover, there exists $i \in S_{\max }(\mathcal{P})$ such that $x_{i}<f(k)$, because otherwise for any $j \in S_{\max }(\mathcal{P})$ we have $x_{j} \geq f(k)$, which implies $\sum_{j \in S_{\max }(\mathcal{P})} x_{j} \geq\left|S_{\max }(\mathcal{P})\right| f(k)$ and $\sum_{j \in S_{\max }(\mathcal{P})} x_{j} \stackrel{x \in X(v, \mathcal{P})}{=} f(|\mathcal{P}|)$ : a contradiction. Hence, there exists $i \in S_{\max }(\mathcal{P})$ such that $x_{i}<f(k)$, and the player $i$ has an incentive to deviate.

\section{References}

[1] Abe T (2018) Stable coalition structures in symmetric majority games: a coincidence between myopia and farsightedness. Theory and Decision 85:353-374

[2] Abe T, Funaki Y (2017) The non-emptiness of the core of a partition function form game. International Journal of Game Theory 46:715-736

[3] Albizuri MJ, Arin J, Rubio J (2005) An axiom system for a value for games in partition function form. International Game Theory Review 7:63-72 
[4] Aumann RJ (1967) A survey of cooperative games without side payments. Essays in Mathematical Economics, Princeton, Princeton University Press

[5] Bloch F, van den Nouweland A (2014) Expectation formation rules and the core of partition function games. Games and Economic Behavior 88:339-353

[6] Bolger E (1989) A set of axioms for a value for partition function games. International Journal of Game Theory 18:37-44

[7] Casajus A (2009) Outside options, component efficiency, and stability. Games and Economic Behavior 65:49-61

[8] Chwe MSY (1994) Farsighted coalitional stability. Journal of Economic Theory 63:299-325

[9] de Clippel G, Serrano R (2008) Marginal contributions and externalities in the value. Econometrica 76:1413-1436

[10] Diamantoudi E, Xue L (2003) Farsighted stability in hedonic games. Social Choice and Welfare 21:39-61

[11] Greenberg J (1994) Coalition structures. Handbook of game theory with economic applications, Chapter 37, Amsterdam, Elsevier

[12] Funaki Y, Yamato T (1999) The core of an economy with a common pool resource: a partition function form approach. International Journal of Game Theory 28:157-171

[13] Hafalir IE (2007) Efficiency in coalition games with externalities. Games and Economic Behavior 61:242-258

[14] Hart S, Kurz M (1983) Endogenous formation of coalitions. Econometrica 51:1047-1064

[15] Kóczy L (2007) A recursive core for partition function form games. Theory and Decision 63:41-51

[16] Kóczy L (2009) Sequential coalition formation and the core in the presence of externalities. Games and Economic Behavior 66:559-565

[17] Kóczy L, Lauwers L (2004) The coalition structure core is accessible. Games and Economic Behavior 48:86-93

[18] Macho-Stadler I, Pérez-Castrillo D, Wettstein D (2007) Sharing the surplus: an extension of the Shapley value for environments with externalities. Journal of Economic Theory 135:339-356

[19] Myerson R (1977) Values of games in partition function form. International Journal of Game Theory 6:23-31

[20] Ray D, Vohra R (1997) Equilibrium binding agreements. Journal of Economic Theory 73:30-78

[21] Shenoy P (1979) On coalition formation: a game theoretical approach. International Journal of Game Theory 8:133-164

[22] Thrall RM (1961) Generalized characteristic functions for n-person games. Proceedings of the Princeton University Conference of Oct. 1961

[23] Thrall RM, Lucas WF (1963) n-Person games in partition function form. Naval Research Logistics Quarterly 10:281-298

[24] von Neumann J, Morgenstern O (1944) Theory of Games and Economic Behavior, Princeton, Princeton University Press

[25] Xue L (1997) Nonemptiness of the largest consistent set. Journal of Economic Theory 73:453-459

[26] Yi SS (1997) Stable coalition structures with externalities. Games and Economic Behavior 20:201- 
